\title{
Utilidad del strain sistólico pico longitudinal bidimensional en pacientes con diagnóstico clínico de infarto del miocardio sin elevación del ST
}

\author{
Santiago Salazar-Marín ${ }^{\mathrm{a}, \mathrm{b}, *}$, Johanna Marcela Valencia ${ }^{\mathrm{a}, \mathrm{b}}$, \\ Oscar Mauricio Hernández-Vásquez ${ }^{\mathrm{C}}$ y Jorge Mario Estrada ${ }^{\mathrm{c}}$
}

\author{
a Instituto Cardiovascular de Risaralda, Pereira, Colombia \\ b Comfamiliar Risaralda, Pereira, Colombia \\ c Universidad CES, Medellín, Colombia
}

Recibido el 31 de octubre de 2016; aceptado el 21 de abril de 2017

Disponible en Internet el 6 de noviembre de 2017

\section{PALABRAS CLAVE \\ Técnicas de imagen cardíaca; \\ Ecocardiografía \\ bidimensional; \\ Síndrome coronario \\ agudo; \\ Enfermedad de arterias coronarias con infarto de miocardio; \\ Contracción miocárdica}

\begin{abstract}
Resumen
Objetivos: evaluar la utilidad del strain sistólico pico longitudinal bidimensional para detectar enfermedad coronaria significativa en pacientes con diagnóstico de infarto agudo del miocardio sin elevación del ST y su capacidad para identificar la arteria responsable del evento agudo. Métodos: estudio observacional descriptivo y transversal realizado entre marzo y noviembre de 2015 en pacientes con diagnóstico clínico de infarto agudo del miocardio sin elevación del ST, a quienes se les evaluó el strain sistólico pico longitudinal bidimensional del ventrículo izquierdo, previo a la angiografía coronaria.

Resultados: se evaluó el strain longitudinal en 28 pacientes que cumplieron con los criterios de selección. Por análisis de curvas ROC, se identificó un punto de corte para el strain global $\geq$ $-18,8 \%$ con sensibilidad del $85 \%$ y especificidad del $75 \%$ para reconocer presencia de enfermedad coronaria angiográficamente significativa. Un punto de corte $\geq-17,8 \%$ de strain global, identificó lesiones significativas con especificidad del 100\%. Para el análisis segmentario se estableció un punto de corte de $\geq 3$ segmentos con strain $\geq-14 \%$, encontrando una sensibilidad de $90 \%$ y una especificidad $87,5 \%$ para diagnóstico de enfermedad coronaria significativa.

Conclusiones: la técnica ecocardiográfica evaluada, aplicada a pacientes con diagnóstico clínico de infarto del miocardio sin elevación del ST, mostró su utilidad para identificar enfermedad coronaria significativa, pero no permitió hallar el vaso culpable del evento agudo.

(C) 2017 Sociedad Colombiana de Cardiología y Cirugía Cardiovascular. Publicado por Elsevier España, S.L.U. Este es un artículo Open Access bajo la licencia CC BY-NC-ND (http:// creativecommons.org/licenses/by-nc-nd/4.0/).
\end{abstract}

\footnotetext{
* Autor para correspondencia.

Correo electrónico: salazar@inscardiovascular.com (S. Salazar-Marín).
} 


\section{KEYWORDS}

Cardiac imaging

techniques; two-dimensional echocardiography; Acute coronary syndrome; Coronary artery diseases with myocardial infarction; Myocardial contraction

\section{Usefulness of two-dimensional longitudinal peak systolic strain in patients with a clinical diagnosis of acute myocardial infarction without ST elevation}

\begin{abstract}
Objectives: To evaluate the usefulness of two-dimensional longitudinal peak systolic strain to detect significant coronary disease in patients with a diagnosis of acute myocardial infarction without ST elevation, and its ability to identify the artery responsible for the coronary event. Methods: A descriptive, observational, and cross-sectional study was conducted between March and November 2015 on patients with a clinical diagnosis of acute myocardial infarction without ST elevation, and on whom the two-dimensional longitudinal peak systolic strain of the left ventricle was evaluated prior to coronary angiography.

Results: The longitudinal strain was evaluated in 28 patients who fulfilled selection criteria. For the ROC curve analysis, a cut-off point of $\geq-18.8 \%$ for the overall strain was identified, with a sensitivity of $85 \%$ and a specificity of $75 \%$ to recognise the presence of angiographically significant coronary disease. A cut-off point of $\geq-17.8 \%$ of overall strain identified significant lesions, with a specificity of $100 \%$. For the segmental analysis, a cut-off point of $\geq 3$ segments with a strain $\geq-14 \%$ was established, finding a sensitivity of $90 \%$ and a specificity of $87.5 \%$ for the diagnosis of significant coronary disease.

Conclusions: The evaluated echocardiographic technique, when applied to patients with a clinical diagnosis of myocardial infarction without ST elevation, was shown to be useful in identifying significant coronary disease, but was unable to find the vessel responsible for the acute event. (c) 2017 Sociedad Colombiana de Cardiología y Cirugía Cardiovascular. Published by Elsevier España, S.L.U. This is an open access article under the CC BY-NC-ND license (http:// creativecommons.org/licenses/by-nc-nd/4.0/).
\end{abstract}

\section{Introducción}

La magnitud del daño miocárdico en el infarto agudo, es un estrecho predictor de mortalidad y eventos cardiovasculares adversos. Los pacientes con infarto de miocardio sin elevación del ST, raramente tienen criterios de reperfusión aguda, a pesar de que algunos pueden sufrir un daño miocárdico extenso si el flujo sanguíneo coronario no es restaurado rápidamente ${ }^{1,2}$. En la actualidad existe discrepancia en cuanto a la utilización de una estrategia agresiva precoz o una diferida en el manejo intervencionista de estos pacientes. Una técnica diagnóstica de fácil aplicación, que permita un diagnóstico rápido, preciso y temprano podría en un futuro ayudar a identificar aquellos con mayor riesgo, en quienes el uso de la terapia intervencionista temprana podría limitar el daño miocárdico, mejorando el pronóstico.

En la secuencia temporal de la cascada isquémica, las alteraciones de la motilidad regional aparecen tempranamente después de la reducción del flujo coronario ${ }^{2}$. La ecocardiografía se convierte en una herramienta diagnóstica extraordinariamente útil en los síndromes coronarios agudos, al permitir detectar dichas alteraciones ${ }^{3}$. Sin embargo, en presencia de infarto de miocardio, dependiendo de su transmuralidad y extensión, las alteraciones contráctiles pueden estar ausentes o no ser detectadas cuando son sutiles, especialmente en el caso de examinadores poco experimentados.

Por la naturaleza incompresible del músculo cardíaco, el acortamiento de los diferentes grupos de fibras miocárdicas durante la sístole ventricular, es responsable del engrosamiento o deformación radial de sus paredes. Cuando se evalúa la contractilidad del ventrículo izquierdo mediante el método de estimación visual, se observa la deformación radial ocasionada por la contracción no solo de las fibras mesoparietales circunferenciales, sino en especial por la contracción de las fibras subepicárdicas y subendocárdicas helicoidales, siendo estas últimas más vulnerables a ser lesionadas por entidades patológicas que afectan el músculo cardíaco, incluida la reducción aguda del flujo coronario ${ }^{4}$, lo cual conduce a deterioro temprano de la deformación longitudinal. Ante compromiso subendocárdico de origen isquémico, sin compromiso de las fibras miocárdicas restantes, puede existir un incremento compensatorio de la contractilidad, principalmente a nivel de las fibras mesoparietales, que eleva el movimiento hacia adentro de la pared, y a su vez potencia la deformación radial y contribuye a la ausencia de disfunción contráctil regional evaluada por el método visual.

Una técnica ecocardiográfica desarrollada en la década pasada, validada por sonomicrometría y resonancia nuclear magnética ${ }^{5}$, permite evaluar la deformación o strain del músculo cardíaco ventricular en los diferentes planos, para lo cual el software del equipo utiliza un algoritmo que demarca automáticamente el miocardio y realiza el seguimiento del movimiento segmentario mediante el método de speckle tracking, cuantificando en forma rápida y precisa el porcentaje de variación en longitud entre el fin de diástole y el fin de sístole de los segmentos evaluados ${ }^{6,7}$. Esta novedosa técnica, proporciona una forma objetiva de cuantificar la función miocárdica global y regional, independiente del ángulo de insonación y parcialmente de movimientos de translación cardiacos, diferenciando entre movimiento miocárdico activo y pasivo, lo cual permite identificar disfunción ventricular sistólica aún en ausencia de trastorno contráctil 
por el método visual, al hallar deterioro de la deformación longitudinal. Su aplicación a pacientes con posible evento coronario agudo, puede incrementar el rendimiento diagnóstico de la ecocardiografía, mejorando su objetividad para detectar disfunción sistólica ventricular.

El propósito de este estudio fue evaluar la utilidad del strain sistólico pico longitudinal bidimensional, en pacientes con diagnóstico clínico de infarto de miocardio sin elevación del ST, en cuanto a su capacidad para identificar enfermedad coronaria significativa y la arteria coronaria responsable del evento agudo, utilizando como patrón de referencia la angiografía coronaria.

\section{Métodos}

Estudio descriptivo observacional de corte transversal de pruebas diagnósticas, que incluyó pacientes $\geq 18$ años, que ingresaron en forma consecutiva al servicio de urgencias de la clínica Comfamiliar Risaralda entre los meses de marzo y noviembre de 2015 con diagnóstico de infarto agudo de miocardio sin elevación del segmento ST. El estudio se efectuó conforme a las reglamentaciones y principios éticos existentes para estudios clínicos de investigación en humanos y fue aprobado por el comité de ética e investigación en salud de Comfamiliar Risaralda, con un riesgo mínimo según la resolución 8430 . Se obtuvo el asentimiento informado de los pacientes incluidos en el estudio.

Los pacientes admitidos cumplieron los siguientes criterios: dolor precordial de 10 o más minutos de duración, ausencia de supradesnivel del ST en el electrocardiograma de superficie, troponina positiva en muestra de suero en las primeras 16 horas del inicio del dolor, coronariografía realizada dentro de las 48 horas siguientes al ingreso, ecocardiografía transtorácica previa con medición y análisis del strain sistólico pico longitudinal bidimensional. Los criterios de exclusión fueron: ventana acústica subóptima, infarto de miocardio previo, cirugía cardíaca previa, supradesnivel del segmento ST (> 0,2 mv en precordiales V1-V3 o > 0,1 $\mathrm{mv}$ en otras derivaciones) en dos o más derivaciones contiguas, bloqueo completo de rama izquierda del haz de His, estimulación ventricular por marcapaso cardíaco, enfermedad valvular cardíaca moderada a severa, miocardiopatías y arritmias cardíacas durante la evaluación ecocardiográfica.

La troponina I utilizada en el estudio fue sensible contemporánea. Su valor se obtuvo utilizando un analizador Cobas e 411 (Roche. Mannheim, Alemania) con calibradores y reactivos específicos para dicho analizador. Se consideró como positivo un valor en suero $>0,16 \mathrm{ng} / \mathrm{ml}$ recomendado por el fabricante, correspondiente al percentil 99 del límite de referencia en individuos sanos. Para la troponina usada, la menor concentración con coeficiente de variación $\leq 10 \%$, fue $0,30 \mathrm{ng} / \mathrm{ml}$ para el grupo control de referencia.

El ecocardiograma transtorácico se realizó con un equipo GE Vivid E9 con XDclear (Vingmed; Horten, Norway) con un transductor con arreglo de fase M5S de 1,5 a 4,6 MHz. Para el cálculo del strain sistólico pico longitudinal, se obtuvieron imágenes en decúbito lateral izquierdo en planos apicales de eje largo, cuatro y dos cámaras registrando tres ciclos consecutivos, con 60 a 80 cuadros por segundo y frecuencia de armónica de $1,7 / 3,3 \mathrm{MHz}$. Las imágenes almacenadas digitalmente fueron analizadas por un solo examinador directamente en el equipo, mediante la técnica AFI (del inglés Automatic Functional Imaging) para obtener los valores de strain en cada uno de los 16 segmentos del ventrículo izquierdo, proporcionando una gráfica en "ojo de buey" con los valores correspondientes al promedio transmural del valor pico de deformación positiva o negativa para cada segmento, durante la sístole ventricular. El valor del strain global se obtuvo al promediar los valores obtenidos en todos los segmentos evaluados. La duración de la sístole ventricular para el estudio, fue definida automáticamente por el equipo utilizando el pico de la onda $\mathrm{R}$ del electrocardiograma y el nadir de la deformación sistólica global mediante las curvas de strain, éste último como sustituto del cierre valvular aórtico. La función sistólica del ventrículo izquierdo se calculó con el método de speckle tracking, definiendo en forma automática los contornos endocárdicos en proyecciones apicales de cuatro y dos cámaras, obteniendo la fracción de eyección según resultado de volúmenes de fin de diástole y sístole. Para evaluar la geometría del ventrículo izquierdo, se calculó el grosor relativo de pared posterior y el índice de masa del ventrículo izquierdo, utilizando las recomendaciones de la Sociedad Americana de Ecocardiografía y la Asociación Europea de Imagen Cardiovascular ${ }^{8}$. Siguiendo esas recomendaciones, la contractilidad segmentaria evaluada por el método visual y definida como normal, hipocinética, acinética o discinética. Se midió la presión arterial sistémica y la frecuencia cardíaca durante el estudio ecocardiográfico. Los resultados de la ecocardiografía se informaron previo a la realización de la angiografía coronaria y en caso de detectarse anormalidad en valores del strain, se incluyó en el informe el territorio miocárdico comprometido.

La aproximación a la identificación de la arteria coronaria responsable del evento agudo, se basó en el modelo de 16 segmentos, que es considerando el más apropiado para evaluar motilidad parietal y perfusión miocárdica mediante ecocardiografía ${ }^{8-10}$; dividiendo el ventrículo izquierdo en tres regiones: basal, medial y apical. El segmento 17 no se incluye en el análisis de strain $^{8}$ y su valor, proporcionado por el equipo, corresponde al promedio de los otros cuatro segmentos apicales. Para propósitos del estudio se consideró como anormal un valor de strain longitudinal pico sistólico segmentario $\geq-14 \%$. Cuando se encontraron valores anormales, se hizo una aproximación a la posible arteria responsable del evento agudo, basándose en la irrigación usual descrita para los diferentes segmentos del ventrículo izquierdo ${ }^{8,9}$ (fig. 1), como se describe a continuación:

- Arteria coronaria descendente anterior (seis segmentos): segmentos basal (1), medio (7) y apical (13) de la pared anterior; segmentos basal (2) y medio (8) anteroseptal; segmento apical (14) del tabique.

- Arteria coronaria derecha (cinco segmentos): segmentos basal (3) y medio (9) del tabique; segmentos basal (4), medio (10) y apical (15) de la pared inferior.

- Arteria coronaria circunfleja (cinco segmentos): segmentos basal (5) y medio (11) de la pared posterior; segmentos basal (6), medio (12) y apical (16) de la pared lateral.

La angiografía coronaria se hizo en las primeras 48 horas posteriores al ingreso, considerando enfermedad coronaria 
A

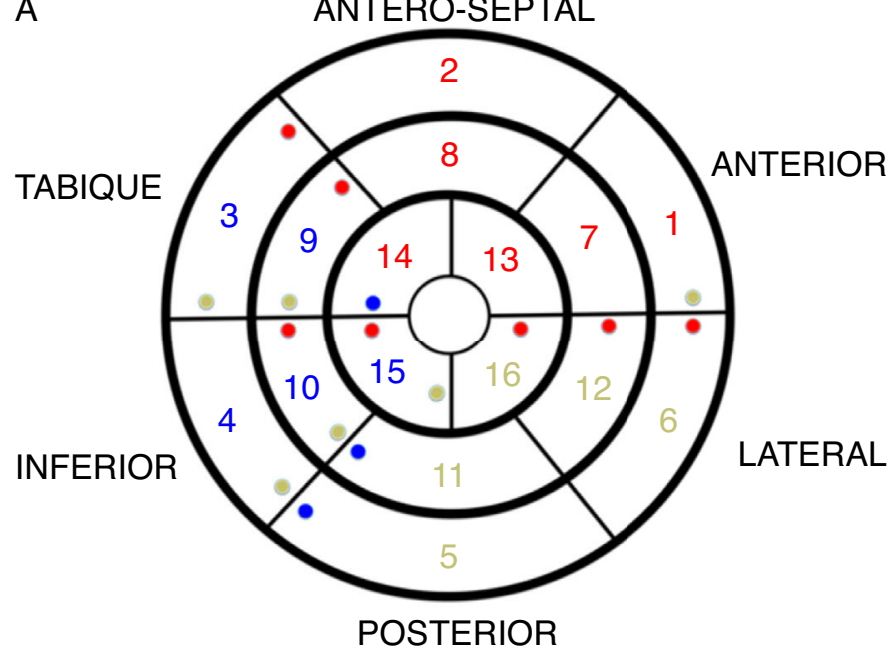

Perfusión usual

Perfusión alternativa
ADA

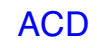

B

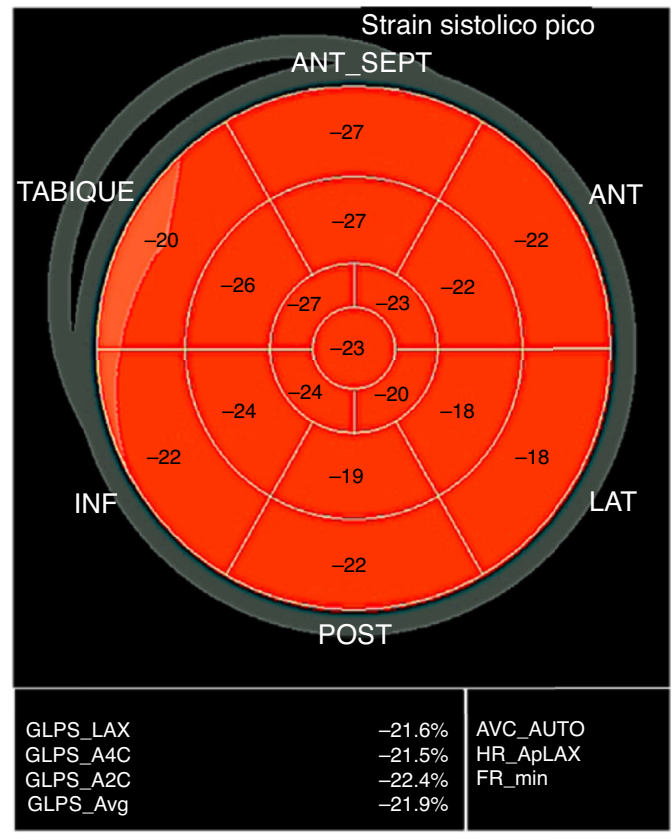

Figura 1 Modelo de 16 segmentos del ventrículo izquierdo. (A) Representación esquemática del mapa polar del strain con la perfusión miocárdica atribuida usualmente a las tres arterias epicárdicas mayores. Los números con su respectivo color, identifican los segmentos usualmente perfundidos por cada una de las arterias. Los puntos en color representan la irrigación menos usual informada para cada segmento. (B) Mapa polar del strain sistólico pico longitudinal bidimensional obtenido en un paciente normal. En la imagen aparece el valor del strain para cada uno de los segmentos. En el recuadro inferior aparece el valor de strain global (GLPS avg -21,9\%) promedio de todos los segmentos.

significativa, una lesión con reducción del lumen arterial $\geq$ $70 \%$ en alguna de las arterias epicárdicas mayores $0 \geq a$ $50 \%$ en el tronco de coronaria izquierda, definiendo oclusión coronaria como flujo TIMI 0 o 1. La morfología angiográfica se usó para definir las lesiones como agudas o crónicas, basándose en hallazgos como disección de la íntima, trombos, ulceración, calcificación y ausencia de circulación colateral.

Se estimó un tamaño de muestra mínimo de 27 pacientes con un nivel de confianza del 95\%, a través del software EPIDAT versión 4.0, utilizando una sensibilidad esperada para el strain longitudinal global no inferior al $70 \%$ con base en reportes de otros estudios y utilizando una prevalencia para la presencia de enfermedad coronaria significativa del $70 \%$ para el grupo poblacional estudiado, según registros institucionales. El diseño de muestreo utilizado fue no probabilístico conformando la muestra consecutivamente y a conveniencia según la consulta y cumplimiento de criterios de inclusión y exclusión.

En el análisis estadístico se consideraron las variables categóricas y cuantitativas que fueron descritas mediante estadística descriptiva, representadas en media y mediana. La variabilidad fue representada por desviaciones estándar o rango intercuartílico según previa evaluación de la forma de distribución. Como prueba de distribución normal sobre variables cuantitativas se utilizó la prueba Shapiro-Wilks considerándose como normal aquellas con $p>0,05$. En el análisis bivariado para identificar diferencias entre los grupos según pruebas de normalidad, se aplicó la prueba t de Student para grupos independientes o no paramétrica como Mann-Whitney. Para los contrastes de hipótesis, se tomó un nivel de significancia de 0,05.
En la estimación de las características operativas de las pruebas, se utilizó un análisis de curvas ROC no paramétrico, calculando el área bajo la curva y determinando puntos de corte para el valor strain longitudinal global y número de segmentos afectados. Se realizó un modelo de regresión logística binaria con el fin de evaluar los puntos de corte seleccionados calculando sensibilidad, especificidad y valores predictivos positivos y negativos. Todos los análisis se hicieron con software STATA, versión 10.0.

Se controló el sesgo de interpretación al realizar la medición e información de la prueba (strain sistólico pico longitudinal) en forma ciega e independiente, sin conocer los resultados obtenidos por el patrón de referencia (angiografía coronaria). El sesgo de información fue controlado al implementar mediciones por parte de profesionales con entrenamiento y experiencia en los procedimientos. El sesgo de verificación parcial de la enfermedad no se presentó ya que todos los pacientes evaluados fueron clasificados mediante el patrón de referencia.

\section{Resultados}

Durante los nueve meses del estudio, veintiocho pacientes cumplieron criterios de selección, el 63,3\% fueron del sexo masculino, la edad promedio fue 58,3 \pm 10 años, encontrándose como factores de riesgo cardiovascular: hipertensión arterial $(53,6 \%)$, diabetes mellitus tipo $2(21,4 \%)$, dislipidemia $(21,4 \%)$, tabaquismo activo $(28,6 \%)$ e hipertrofia ventricular izquierda (25\%). Durante el estudio ecocardiográfico, la presión arterial sistólica fue 133,8 $\pm 15,6 \mathrm{~mm} \mathrm{Hg}$, 
Tabla 1 Comparación de resultados de fracción de eyección y strain longitudinal, en pacientes con y sin lesiones coronarias angiográficamente significativas. La prueba de comparación utilizada se basó en el test de normalidad y según resultado se utilizaron pruebas paramétricas y no paramétricas

\begin{tabular}{llll}
\hline & Con lesión significativa $(\mathrm{n}=20)$ & Sin lesión significativa $(\mathrm{n}=8)$ & Valor $\mathrm{p}$ \\
\hline${\text { Fracción de eyección VI }(\%)^{*}}_{\text {Strain longitudinal global }(\%)^{* *}}$ & $58(4,5)$ & $60(1)$ & 0,28 \\
Número de segmentos afectados $^{* * *}$ & $6(5,5)$ & $-19,5(1,4)$ & 0,0004 \\
\hline
\end{tabular}

" Se presentan los resultados como la media (DE); la prueba de normalidad Shapiro-Wilks no fue significativa.

** Se presentan los resultados como la mediana (IRQ); la prueba de normalidad Shapiro-Wilks fue significativa.

con diastólica de $75,5 \pm 8,3 \mathrm{~mm} \mathrm{Hg}$ y frecuencia cardiaca de $73 \pm 7$ latidos por minuto. Los valores de fracción de eyección y strain global longitudinal pico sistólico del ventrículo izquierdo, discriminados según presencia o ausencia de lesiones coronarias angiográficamente significativas, se muestran en la tabla 1. Las diferencias en la distribución de los valores de strain por segmentos entre los dos grupos de estudio, mostró sistemáticamente distribuciones en un rango $\geq-14 \%$, con mayor variabilidad en el grupo de pacientes con lesión angiográficamente significativa, como se observa en la figura 2.

El índice de masa ventricular izquierda promedio fue mayor en mujeres $(96,7 \pm 32,7)$ que en los hombres $(88,6 \pm 25,3)$ debido al predominio de hipertrofia moderada a severa en ellas. En 7 de 15 pacientes con antecedente de hipertensión arterial, se encontró hipertrofia ventricular izquierda concéntrica. En los restantes el índice de masa ventricular izquierda fue normal, sin hallar diferencia significativa en los valores de strain longitudinal global, entre pacientes con y sin hipertrofia ventricular izquierda $(-20,5$ a $-14,3 \%$, media $-16,1 \%$ vs. $-20,9$ a $-13,2 \%$, media $-17,4 \%$, $p=0,352)$.

Seis pacientes tenían antecedentes de diabetes mellitus tipo 2 y en cuatro de ellos con fracción de eyección normal, se encontró uno con strain global bajo (-13,2\%) y strain segmentario anormal en el territorio de las tres arterias epicárdicas y enfermedad coronaria significativa de tres vasos; otro con strain global bajo $(-14,2 \%)$ y strain segmentario anormal en el territorio de la arteria coronaria derecha y enfermedad coronaria significativa de tres vasos y en los 2 restantes los valores de strain global y segmentario normal fueron normales, encontrándose enfermedad coronaria significativa en uno de ellos con compromiso de circunfleja y arteria coronaria derecha. De veinte $(71,4 \%)$ pacientes con enfermedad coronaria angiográficamente significativa, en $16(80 \%)$ se encontró enfermedad multivaso (dos vasos, el $45 \%$ y tres vasos el $35 \%$ ) y en $7(43,7 \%)$ de esos 16 , los hallazgos angiográficos no permitieron identificar la posible

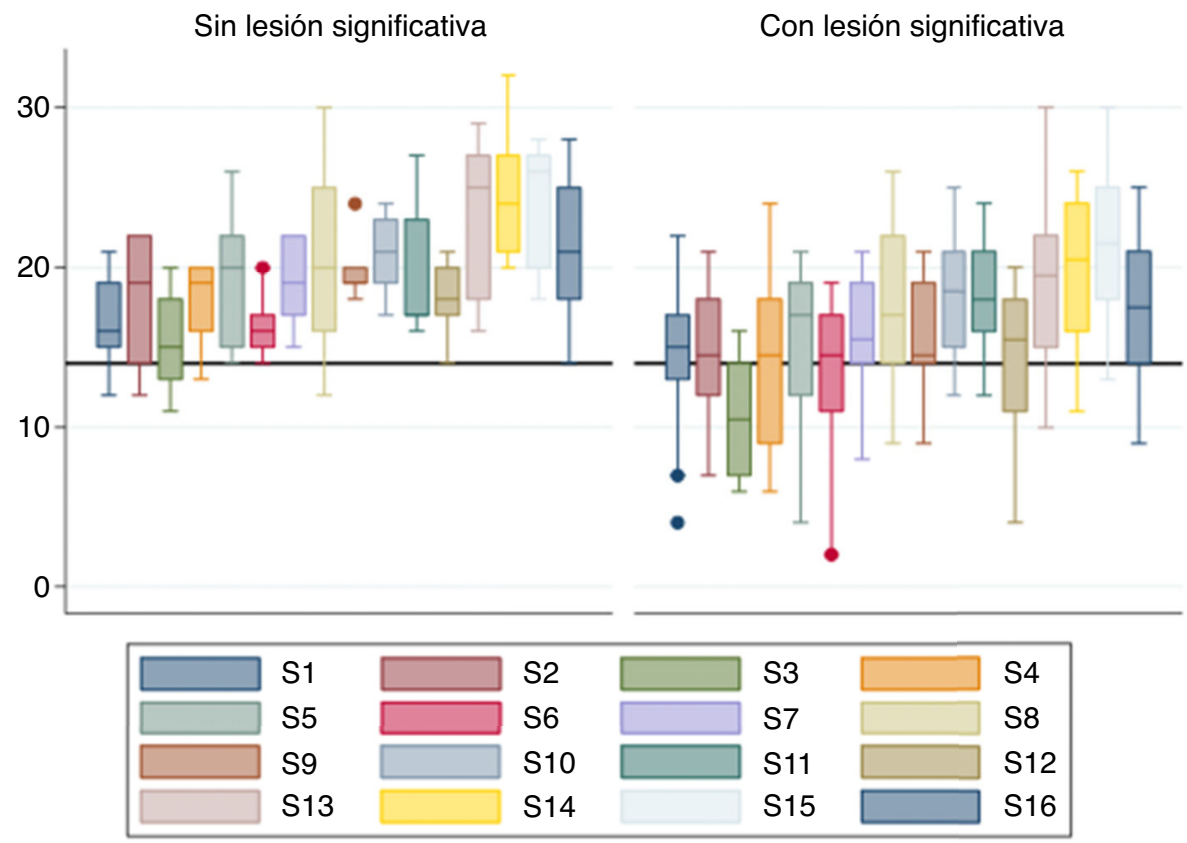

Figura 2 Distribución de valores de strain longitudinal por segmentos entre pacientes con y sin enfermedad coronaria angiográficamente significativa. Las barras en diferente color, permiten identificar cada uno de los dieciséis segmentos (S) del ventrículo izquierdo según el acercamiento de perfusión para cada una de las tres arterias epicárdicas mayores en relación con el modelo de los dieciséis segmentos de la figura 1. 
arteria responsable del evento agudo, generando un sesgo que no permitió evaluar la utilidad del strain segmentado para dicho propósito.

Las características operativas del strain sistólico pico longitudinal bidimensional para identificar lesiones coronarias angiográficamente significativas, se observan en la figura 3. El análisis de la curva ROC para el valor de strain global identificó un punto de corte $\geq-18,8 \%$ como el de mayor sensibilidad y especificidad (fig. 3a), con un área bajo la curva de 0,89 (IC95\%, 0,51-0,99), razones de probabilidad positiva de 3,4 y negativa de 0,2 , con una capacidad predictiva para la identificación de lesiones coronarias significativas del $89 \%$ y un intervalo de confianza amplio relacionado con el bajo tamaño de la muestra. El análisis de la curva ROC para número de segmentos afectados con valor de strain $\geq-14 \%$, encontró que el punto de corte con mayor sensibilidad y especificidad fue $\geq 3$ segmentos afectados, con un área bajo la curva de 0,89 (IC95\%, 0,78-0,99) (fig. 3b), es decir con clasificación correcta de los casos de un $89 \%$, con razón de probabilidad positiva 7,2 y negativa 0,11 . Para

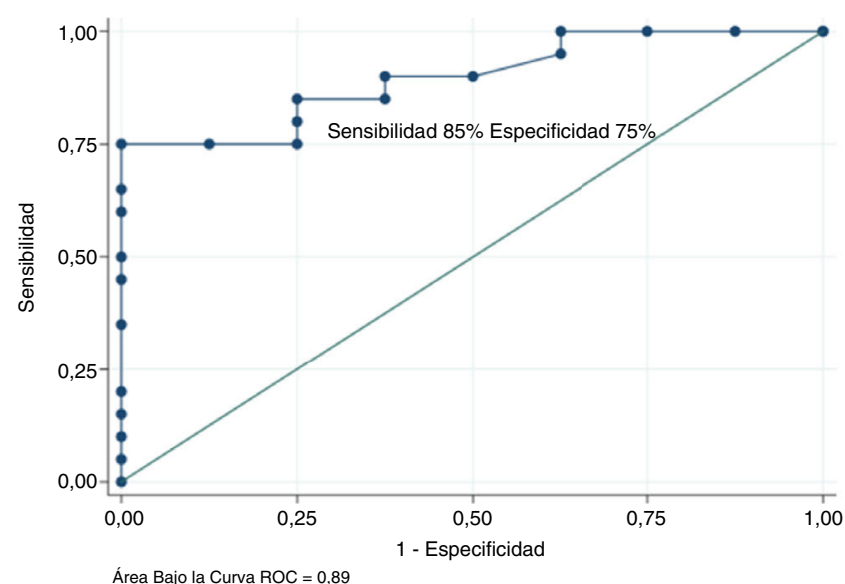

Strain global (a)

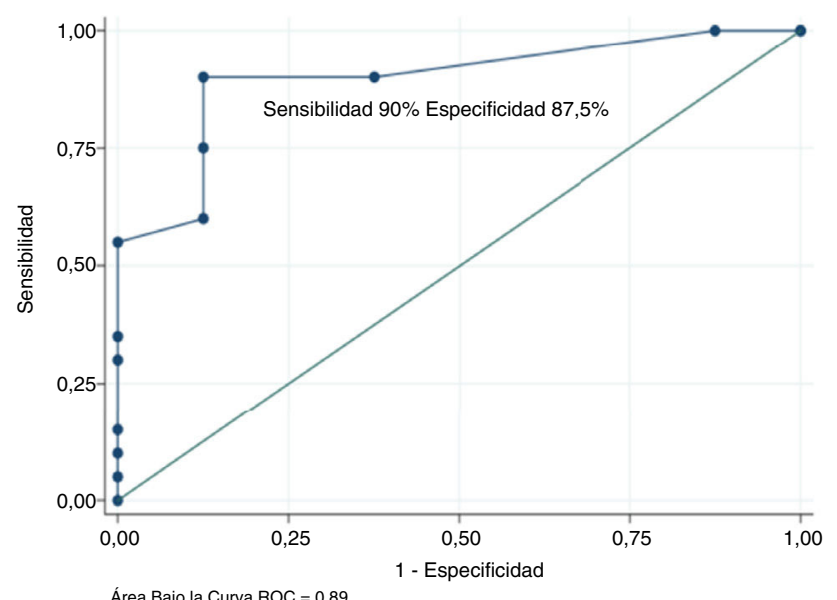

Strain segmentario (b)
Tabla 2 Características operativas diagnósticas para punto de corte en strain longitudinal

\begin{tabular}{lll}
\hline & $\begin{array}{l}\text { Strain } \\
\text { global } \geq \\
-18,8 \%\end{array}$ & $\begin{array}{l}\text { Strain } \geq-14 \% \\
\text { en } \geq 3 \\
\text { segmentos }\end{array}$ \\
\hline Sensibilidad (\%) & 85 & 90 \\
Especificidad (\%) & 75 & 87,5 \\
Valor predictivo positivo (\%) & 89,5 & 94 \\
Valor predictivo negativo (\%) & 66,7 & 77,8 \\
Falsos positivos (\%) & 25 & 12,5 \\
Falsos negativos (\%) & 15 & 10 \\
\hline
\end{tabular}

clasificar las variables por los puntos de corte mencionados para strain longitudinal global y número de segmentos afectados mediante regresión logística binaria, se calcularon las características operativas de utilidad diagnóstica como lo muestra la tabla 2 .

\begin{tabular}{|c|c|c|c|c|c|}
\hline Strain & Sensibilidad & Especificidad & AUC & RP + & RP - \\
\hline$\geq-20,5$ & 1002 & 57 & 81 &, 3 & 0,000 \\
\hline$\geq-20,4$ & 100 & 37 & 82 & 1,6 & 0,000 \\
\hline$\geq-20,0$ & 95 & 37 & 78 & 1,5 & 0,133 \\
\hline$\geq-19,2$ & 90 & 50 & 78 & 1,5 & 0,200 \\
\hline$\geq-19,1$ & 90 & 62 & 82 & 2,4 & 0,160 \\
\hline$\geq-19,0$ & 85 & 62 & 78 & 2,3 & 0,240 \\
\hline$\geq-18,8$ & 85 & 75 & 89 & 3,4 & 0,200 \\
\hline$\geq-18,4$ & 80 & 75 & 78 & 3,2 & 0,267 \\
\hline$\geq-18,2$ & 75 & 75 & 75 & 3,5 & 0,333 \\
\hline$\geq-18,0$ & 75 & 87 & 78 & 6 & 0,286 \\
\hline$\geq-17,8$ & 75 & 100 & 82 & & 0,250 \\
\hline$\geq-16,2$ & 65 & 100 & 75 & & 0,350 \\
\hline$\geq-15,3$ & 60 & 100 & 71 & & 0,400 \\
\hline
\end{tabular}

\begin{tabular}{|c|c|c|c|c|c|}
\hline $\begin{array}{c}\text { No segmentos } \\
\geq-14 \%\end{array}$ & Sensibilidad & Especificidad & AUC & RP + & RP - \\
\hline$\geq 1$ & 1001 & 2,57 & 51 &, 4 & 0,000 \\
\hline$\geq 2$ & 90 & 62,5 & 82 & 2,4 & 0,160 \\
\hline$\geq 3$ & 90 & 87,5 & 89 & 7,2 & 0,114 \\
\hline$\geq 4$ & 75 & 87,5 & 78 & 6 & 0,285 \\
\hline$\geq 5$ & 60 & 87,5 & 68 & 4,8 & 0,457 \\
\hline$\geq 6$ & 55 & 100 & 68 & & 0,450 \\
\hline$\geq 7$ & 35 & 100 & 53 & & 0,650 \\
\hline
\end{tabular}

Figura 3 Curvas ROC de valor strain longitudinal global (a) y número de segmentos afectados (b) en la identificación de lesiones coronarias angiográficamente significativas. AUC, área bajo la curva; RP +, razón de probabilidad positiva; RP -, razón de probabilidad negativa. 


\section{Discusión}

Se ha encontrado que la determinación ecocardiográfica del strain sistólico pico longitudinal bidimensional, es mejor que la evaluación del desplazamiento y engrosamiento parietal, para discriminar entre el miocardio infartado y el normal, por lo que su aplicación en pacientes con sospecha de un evento coronario agudo puede ser de gran utilidad ${ }^{11-13}$. En pacientes con infarto agudo de miocardio sin elevación del ST, esta nueva técnica puede predecir su tamaño, habiéndose encontrando que un strain longitudinal global $\geq-13,8 \%$ identifica en forma segura los pacientes con infarto significativo ( $\geq$ al $12 \%$ del total del volumen miocárdico $)^{2}$; proporcionando además información pronóstica, como se encontró en un estudio prospectivo que incluyó 849 pacientes, donde en presencia de fracción de eyección normal o relativamente conservada (> 40\%), un valor de strain longitudinal global $>-14 \%$ en medición realizada dentro de las 48 horas del ingreso, identificó aquellos de alto riesgo en el seguimiento a 30 meses para el punto final compuesto de muerte y falla cardíaca (hazard ratio [HR] 3,21\%), muerte cardíaca (hazard ratio [HR]12,7\%.) y falla cardíaca (hazard ratio [HR] $5,31 \%)^{12}$. Además, como se confirmó en este estudio, esta nueva técnica ecocardiográfica tiene utilidad diagnóstica, al revelar la presencia de enfermedad coronaria angiográficamente significativa en pacientes con infarto agudo de miocardio sin elevación del ST y función sistólica global normal o levemente comprometida, incluso en ausencia de trastornos de la contractilidad segmentaria por el método de estimación visual.

En este estudio se identificó un punto de corte óptimo para el strain longitudinal global $\geq-18,8 \%$, con sensibilidad del $85 \%$ para detectar aquellos con lesiones coronarias significativas, con valor predictivo positivo del $89,5 \%$, especificidad del $75 \%$ y valor predictivo negativo del $66,7 \%$. Debido a que este punto de corte carece de la especificidad necesaria, su determinación debe reservarse para casos donde existe alto índice de sospecha de evento coronario agudo. Al modificar el punto de corte de strain longitudinal global $a \geq-17,8 \%$, se pudo identificar correctamente a todos los pacientes con lesiones coronarias significativas, con una sensibilidad del $75 \%$, hallazgo que puede explicarse por los valores de strain encontrados en pacientes con lesiones coronarias significativas comparados con aquellos sin lesiones significativas $(-16,0 \pm 2,3 \%$, IC $-13,2 \%$ a $-20,4 \%$ vs $-19,6 \pm 1,1 \%$, IC $-18 \%$ a $-20,9 \%, p=0,0004)$. Publicaciones previas $^{10}$, han reportado que, de manera aislada, los valores del strain longitudinal global no permiten discriminar entre presencia o ausencia de enfermedad coronaria significativa, debido a la superposición entre los valores reportados para individuos normales, con los encontrados en pacientes con lesiones coronarias significativas. Pese a que en este estudio se encontró un punto de corte de strain longitudinal global útil para identificar aquellos con enfermedad coronaria significativa, este resultado debe verse con cautela debido al pequeño número de pacientes evaluados, especialmente si se considera que el valor de corte encontrado, se superpone con valores informados para la población normal, como se observa al comparar los valores hallados en el presente estudio, con los encontrados en un meta-análisis recientemente publicado que incluyó 24 estudios con un total de
2.597 sujetos, que reportó un valor normal de strain longitudina lsistólico pico global que fluctuaba entre $-22,1 \%$ y $-15,9 \%$ (promedio $-19,7 \%$, IC 95\%, - $20,4 \%$ a $-18,9 \%)^{3,14}$.

El análisis del strain longitudinal segmentario, encontró que un valor $\geq-14 \%$ en 3 o más segmentos del territorio de una arteria epicárdica mayor, permitió identificar enfermedad coronaria significativa con sensibilidad del $90 \%$, especificidad del $87,5 \%$ y valor predictivo positivo y negativo de 94 y 77,8\%, respectivamente. En 3 pacientes se encontró hipocinesia segmentaria mediante estimación visual, encontrando valores de strain anormal para dichos segmentos entre $-12 \%$ a $-4 \%$, comparable con valores de $-12 \%$ a $2 \%$ informados previamente para segmentos hipocinéticos ${ }^{13}$. Otros autores han informado que un strain longitudinal segmentario $\geq-14 \%$ hallado en cuatro o más segmentos adyacentes, en pacientes con infarto agudo de miocardio sin elevación del ST, permitía identificar oclusión coronaria aguda con sensibilidad del $85 \%$ y especificidad del $70 \%{ }^{1}$, hallazgo que no pudo ser confirmado en el presente estudio, debido a que se encontraron $8(28,5 \%)$ pacientes con oclusión de alguno de los vasos epicárdicos mayores, todos con hallazgos angiográfícos de oclusión crónica, hecho que explicaría el haber hallado solo 2 de ellos con un strain longitudinal segmentario anormal $(\geq-14 \%$ ) comprometiendo tres segmentos del territorio de la arteria epicárdica ocluida. El valor anormal de strain longitudinal segmentario encontrado en los pacientes con oclusión crónica, podría explicarse por disfunción regional contráctil isquémica, sin que el vaso ocluido fuese el responsable del evento agudo. En los 6 pacientes restantes con oclusión coronaria, el strain normal podría explicarse por ausencia de disfunción miocárdica isquémica.

En este estudio, en 8 pacientes $(28.5 \%)$ con troponina positiva con valores de 0,214 a $1,08 \mathrm{ng} / \mathrm{ml}$, no se halló enfermedad coronaria angiográficamente significativa. Como bien se sabe, una troponina positiva no es sinónimo de infarto agudo de miocardio, pudiendo encontrarse un valor elevado en variadas condiciones clínicas cardíacas o no cardíacas, agudas o crónicas ${ }^{15-19}$. En 4 (14,3\%) pacientes se encontró una explicación clínica para el valor de troponina elevado: miopericarditis aguda, taquicardia supraventricular, puente intramiocárdico y flujo lento intracoronario. En los cuatro $(14,3 \%)$ pacientes restantes con coronarias normales, no se encontró una explicación para elevación de troponina, acercándose a la frecuencia de infarto de miocardio con coronarias normales descrita en la literatura, que fluctúa entre 8 y $12 \%{ }^{20}$. Estos casos pueden explicarse por otros mecanismos: lisis espontánea del coágulo en las primeras horas $^{1,20}$, vasoespasmo coronario transitorio ${ }^{20,21}$, expansión excéntrica de placa ateroesclerótica no detectable por coronariografía, disfunción microvascular o embolización coronaria $^{20}$. Otra posibilidad a considerar específicamente en este estudio como causa de troponina positiva y coronarias normales, puede relacionarse con el coeficiente de variación para la troponina utilizada ${ }^{15}$, con una imprecisión informada por el fabricante $\leq 10 \%$ para una concentración mínima de $0,30 \mathrm{ng} / \mathrm{ml}$, pero no para el valor de corte utilizado en el estudio $>0,16 \mathrm{ng} / \mathrm{ml}$ (correspondiente al percentil 99 del límite normal de referencia), característica que puede generar a su vez una imprecisión diagnóstica de magnitud no cuantificable, pero de todos modos $\geq$ al $10 \%$. Raramente después de una investigación clínica exhaustiva, 
los valores de troponina elevados no pueden ser explicados por alguna de las condiciones mencionadas y estos casos pueden ser considerados como verdaderos falsos positivos relacionados usualmente con la presencia de anticuerpos heterófilos que interfieren su cuantificación, al unirse con los anticuerpos de captura y conjugación que constituyen el ELISA, simulando detección de troponina ${ }^{16}$.

\section{Limitaciones}

Por el número relativamente pequeño de pacientes incluidos en el estudio, los resultados deben ser interpretados con cautela y no pueden considerarse representativos de la población total de infarto agudo de miocardio sin elevación del ST. Igualmente, no pueden ser considerados representativos para todas las máquinas de ultrasonido, debido a que a pesar de informarse valores de strain longitudinal global comparables entre ellas ${ }^{14,22}$, la correlación para valores segmentarios es débil, especialmente en segmentos basales ${ }^{22}$.

La definición automática de la sístole utilizada en el estudio, se ha encontrado subóptima en presencia de condiciones como disfunción sistólica regional significativa y de trastornos de conducción intraventricular ${ }^{23}$, de modo que estos resultados no son aplicables a pacientes con dichas condiciones fisiopatológicas; en estos casos, se recomienda la medición del cierre valvular aórtico utilizando la señal de doppler espectral, para una correcta definición del fin de la sístole.

Un factor limitante mayor del estudio, fue la presencia de enfermedad coronaria multivaso en un alto porcentaje de pacientes con enfermedad significativa, que en ausencia de hallazgos angiográficos orientadores para identificar el vaso responsable del evento agudo en cerca de la mitad de ellos, generó un sesgo que no permitió evaluar la utilidad del strain segmentario para dicho propósito. En este punto se deben considerar dos limitantes adicionales relacionadas con la identificación de los segmentos miocárdicos responsables de la liberación de troponina y su relación con el vaso responsable del evento agudo: la primera, se relaciona con la perfusión diferente a la usual, que puede encontrarse en varios segmentos del ventrículo izquierdo ${ }^{8,9,24}$, donde incluso algunos de ellos pueden tener irrigación dual (fig. 1), pudiendo condicionar inexactitud al momento de correlacionar la irrigación de una arteria epicárdica con determinados segmentos; la segunda, se relaciona con la utilización en el estudio de técnicas poco fiables para localizar topográficamente la zona del infarto agudo, como la ecocardiografía y la angiografía coronaria. Es conocido que la mayoría de episodios coronarios agudos tienen su origen en la trombosis de una placa vulnerable no obstructiva, en muchos casos no significativa ${ }^{25}$, y que existe la posibilidad de que un mismo paciente tenga varias placas rotas, aunque sólo una de ellas sea la causante de la manifestación clínica ${ }^{26}$. Esta inestabilidad coronaria generalizada con activación y complicación de múltiples placas, dificulta la localización de los segmentos responsables de la elevación de la troponina y su correlación con la arteria responsable del evento agudo cuando se utilizan técnicas inexactas para dicho propósito, más aún, en presencia de enfermedad coronaria plurivascular, como ocurrió en el presente estudio.

\section{Conclusiones}

El strain sistólico pico longitudinal bidimensional, en pacientes con diagnóstico clínico de infarto agudo de miocardio sin elevación del ST, permite identificar la presencia de lesiones coronarias significativas al reconocer la disfunción miocárdica segmentaria provocada por la isquemia regional, incluso en ausencia de trastornos de contractilidad del ventrículo izquierdo identificados por el método de estimación visual. Los resultados de este estudio apoyan la importancia de esta nueva técnica ecocardiográfica en la evaluación diagnóstica de este grupo de pacientes, por cuanto su utilización rutinaria podría contribuir a estratificar el riesgo y orientar el manejo. Debido a que se ha encontrado una variación significativa en los valores de strain longitudinal para los diferentes segmentos del ventrículo izquierdo tanto en sujetos sanos ${ }^{27}$ como en aquellos con enfermedad coronaria ${ }^{4}$, podría ser más apropiado determinar puntos de corte para valores de strain por segmentos o regiones, buscando mejorar la exactitud diagnóstica del método.

\section{Responsabilidades éticas}

Protección de personas y animales. Los autores declaran que los procedimientos seguidos se conformaron a las normas éticas del comité de experimentación humana responsable y de acuerdo con la Asociación Médica Mundial y la Declaración de Helsinki.

Confidencialidad de los datos. Los autores declaran que han seguido los protocolos de su centro de trabajo sobre la publicación de datos de pacientes.

Derecho a la privacidad y consentimiento informado. Los autores declaran que en este artículo no aparecen datos de pacientes.

\section{Conflicto de intereses}

Ninguno.

\section{Agradecimientos}

A los pacientes por permitir el acceso a los datos.

\section{Bibliografía}

1. Eek C, Grenne B, Brunvand H, Aakhus S, Endresen K, Smiseth O, et al. Strain echocardiography predicts acute coronary occlusion in patients with non-ST-segment elevation acute coronary syndrome. Eur J Echocardiogr. 2010;11:501-8.

2. Eek C, Grenne B, Brunvand H, Aakhus S, Endresen K, Hol PK, et al. Strain echocardiography and wall motion score index predicts final infarct size in patients with non-ST-segmentelevation myocardial infarction. Circ Cardiovasc Imaging. 2010;3:187-94.

3. Ahumada S, Restrepo G. Ecocardiografía en infarto agudo del miocardio. Rev Colomb Cardiol. 2015;21:164-73.

4. Choi JO, Cho SW, Song YB, Cho SJ, Song BG, Lee SC, et al. Longitudinal $2 \mathrm{D}$ strain at rest predicts the presence of left main and 
three vessel coronary artery disease patients without regional wall motion abnormality. Eur J Echocardiogr. 2009;10:695-701.

5. Amundsen BH, Helle-Valle T, Edvardsen T, Torp H, Crosby J, Lyseggen E, et al. Noninvasive myocardial strain measurement by speckle tracking echocardiography: validation against sonomicrometry and tagged magnetic resonance imaging. J Am Coll Cardiol. 2006;47:789-93.

6. Gorcsan J, Tanaka H. Echocardiographic assessment of myocardial strain. J Am Coll Cardiol. 2011;58:1401-13.

7. Valiente-mustelier J, Rodríguez-londres J, García Fernández R, Cabrera Rego JO, Coutin Marie G, Valiente Turro A. Validez diagnóstica de los parámetros de deformación miocárdica en el diagnóstico de enfermedad arterial coronaria significativa. Rev Cubana Cardiol Cir Cardiovasc. 2012;18:100-5.

8. Lang RM, Badano LP, Mor-Avi V, Afilalo J, Armstrong A, Ernande $L$, et al. Recommendations for cardiac chamber quantification by echocardiography in adults: an update from the American Society of Echocardiography and the European Association of Cardiovascular Imaging. J Am Soc Echocardiogr. 2015;28:1-39.

9. Cerqueira MD, Weissman NJ, Dilsizian V, Jacobs AK, Kaul S, Laskey WK, et al. Standardized myocardial segmentation and nomenclature for tomographic imaging of the heart. A statement for healthcare professionals from the Cardiac Imaging Committee of the Council on Clinical Cardiology of the American Heart Association. Circulation. 2002;105:539-42.

10. Norum IB, Ruddox V, Edvardsen T, Otterstad JE. Diagnostic accuracy of left ventricular longitudinal function by speckle tracking echocardiography to predict significant coronary artery stenosis. A systematic review. BMC Medical Imaging. 2015;15: $1-12$.

11. Gotte MJ, van Rossum AC, Twisk JWR, Kuijer JPA, Marcus JT, Visser CA. Quantification of regional contractile function after infarction: strain analysis superior to wall thickening analysis in discriminating infarct from remote myocardium. J Am Coll Cardiol. 2001;37:808-17.

12. Ersboll M, Valeur N, Mogensen UM, Andersen MJ, Meller JE, Velazquez EJ, et al. Prediction of all-cause mortality and heart failure admissions from global left ventricular longitudinal strain in patients with acute myocardial infarction and preserved left ventricular ejection fraction. J Am Coll Cardiol. 2013;61:2365-73.

13. Takahashi M, Harada N, Isozaki Y, Lee K, Yajima R, Kataoka A, et al. Efficiency of quantitative longitudinal peak systolic strain values using automated function imaging on transthoracic echocardiogram for evaluating left ventricular wall motion: new diagnostic criteria and agreement with naked eye evaluation by experience. Int J Cardiol. 2013;167:1625-31.

14. Yingchoncharoen T, Agarwal S, Popovic Z, Marwick TH. Normal ranges of left ventricular strain: a meta-analysis. J Am Soc Echocardiogr. 2013;26:185-91.
15. Jarolim P. High sensitivity cardiac troponin assays in the clinical laboratories. Clin Chem Lab Med. 2015;53:635-52.

16. Agewall S, Giannitsis E, Jernberg T, Katus H. Troponin elevation in coronary vs. non-coronary disease. Eur Heart J. 2011;32:404-11.

17. Schwarz ER, Klues HJ, vom Dahl J, Klein I, Krebs W, Hanrath P. Functional characteristics of myocardial bridging. A combined angiographic and intracoronary doppler flow study. Eur Heart J. 1997; 18:434-42.

18. Echeverry EJ, Murillo NE, Villadiego JS, Ochoa JA, Velásquez JG. Puentes miocárdicos como causa de infarto agudo del miocardio. Reporte de una serie de once casos y revisión de la literatura. Rev Colomb Cardiol. 2013;20:240-5.

19. Uribe CE, Solano E, Estrada G, Calderón LI, Castro P, Hurtado $\mathrm{E}$, et al. Fenómeno de flujo coronario lento: registro de 140 pacientes en la clínica Abbott Shaio. Rev Colomb Cardiol. 2007;14:87-92.

20. Cortell A, Sanchis J, Bodí V, Núñez J, Mainar L, Pellicer M, et al. Infarto de miocardio sin elevación del ST con coronarias normales: predictores y pronóstico. Rev Esp Cardiol. 2009;62:1260-6.

21. Ong P, Athanasiadis A, Borgulya G, Voehringer M, Sechtem U. 3-Year follow-up of patients with coronary artery spasm as cause of acute coronary syndrome: the CASPAR (Coronary Artery Spasm in Patients with Acute Coronary Syndrome) study followup. J Am Coll Cardiol. 2011;57:147-52.

22. Patrianakos AP, Zacharaki AA, Kalogerakis A, Solidakis G, Parthenakis FI, Vardas PE. Two-dimensional global and segmental longitudinal strain: are the results from software in diferente high-end ultrasound systems comparable. Echo Res Pract. 2015;2:29-39.

23. Mada RO, Lysyansky P, Daraban AM, Duchenne J, Voigt JU. How to define end-diastole and end- systole?: Impact of timing on strain measurements. JACC Cardiovasc Imaging. 2015;8:148-57.

24. Ortiz-Pérez JT, Rodríguez J, Meyers SN, Lee DC, Davidson C, Wu E. Correspondence between the 17-Segment model and coronary arterial anatomy using contrast-enhanced cardiac magnetic resonance imaging. J Am Coll Cardiovasc Imaging. 2008;1:282-93.

25. Vilariño JO, Esper R, Badimón JJ. Fisiopatología de los síndromes coronarios agudos. Tres paradigmas para un nuevo dogma. Rev Esp Cardiol. 2004;4 Supl:13G-24G.

26. Ambrose JA, Tannenbaum MA, Alexopoulos D, Hjemdahl-Monsen CE, Leavy J, Weiss M, et al. Angiographic progression of coronary artery disease and the development of myocardial infarction. $J$ Am Coll Cardiol. 1988;12:56-62.

27. Marwick TH, Leano RL, Brown J, Sun JP, Hoffmann R, Lysyansky $P$, et al. Myocardial strain measurement with 2-dimensional speckle-tracking echocardiography. definition of normal range. JACC Cardiovasc Imaging. 2009;2:80-4. 